\title{
Auditory and vestibular symptoms after COVID-19 infection: a preliminary Brazilian report
}

\author{
Rubens Jonatha dos Santos Ferreira ${ }^{1}$ \\ https://orcid.org/0000-0003-3404-6210 \\ Hionara Nascimento Barboza ${ }^{1}$ \\ https://orcid.org/0000-0003-2539-618X \\ Ana Loísa de Lima e Silva Araújo' \\ https://orcid.org/0000-0002-9297-5969 \\ Scheila Farias de Paiva ${ }^{2}$ \\ https://orcid.org/0000-0002-5426-7873 \\ Marine Raquel Diniz da Rosa ${ }^{1}$ \\ https://orcid.org/0000-0003-0920-2234
}

Universidade Federal da Paraíba - UFPB, Departamento de Fonoaudiologia, João Pessoa, Paraíba, Brasil.

2 Universidade Federal de Sergipe - UFSE, Departamento de Fonoaudiologia,

Aracaju, Sergipe, Brasil.

Conflict of interests: Nonexistent

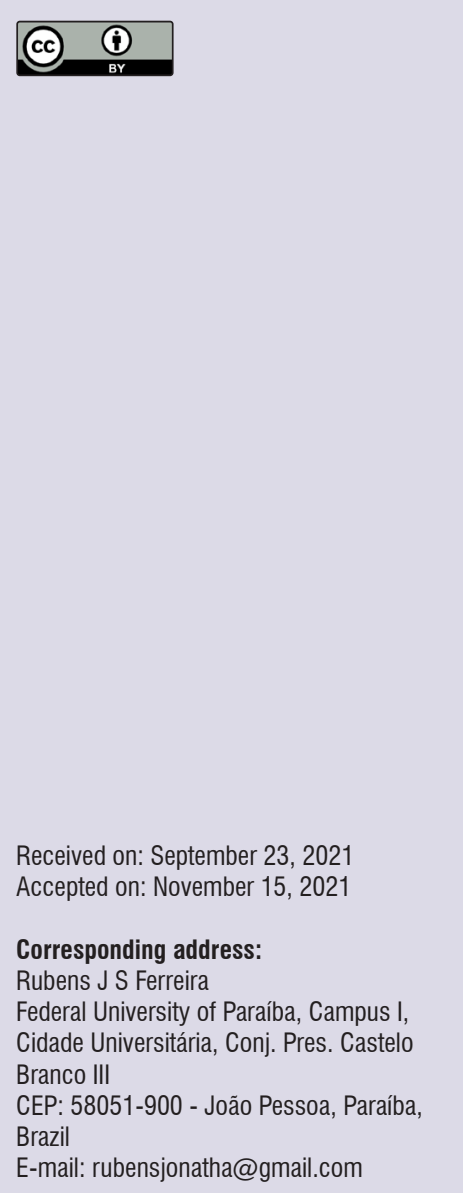

\section{ABSTRACT}

Purpose: to present preliminary data on the influence of COVID-19 on the appearance and/or worsening of auditory and vestibular symptoms in the population of a Brazilian state.

Methods: an observational, descriptive and quantitative approach of research, carried out from September 2020 to May 2021, in Paraíba, Northeastern Brazil. The research consisted of a self-report questionnaire on the manifestation of COVID-19, general post-infection symptoms, and audio-vestibular symptoms before and after COVID-19, posted on social media using the snowball technique.

Results: the sample consisted of 173 people, $89 \%$ reporting symptomatic manifestations of COVID-19 with home treatment, $6.9 \%$ being asymptomatic, and $4 \%$ symptomatic with hospital treatment. A decrease in smell was reported by 126 patients, headache by 76 , tinnitus by 76 , and dizziness by 72 , after infection by COVID-19. An increase in audio-vestibular symptoms was observed, affecting even those who did not present them before COVID.

Conclusions: the preliminary results of the research point to a high occurrence and worsening of auditory and vestibular symptoms, following COVID-19.

Keywords: Tinnitus; Dizziness; COVID-19; Pathological Conditions, Signs and Symptoms; Hearing 


\section{INTRODUCTION}

The new coronavirus disease has affected the world population since the end of 2019 , becoming a pandemic in January 2020. In June 2021, estimates by the World Health Organization (WHO) pointed to around 173 million infected people worldwide, and an average number of 3.72 million deaths, due to complications triggered by this virus ${ }^{1}$. In Brazil, reports from the Ministry of Health showed that more than 16 million Brazilians were infected and the number of deaths exceeded 474,000 in the same period ${ }^{2}$.

The SARS-CoV-2 virus, from the beta-coronavirus family, is easy to enter host cells and spread in the organism due to its structural and reproduction characteristics. These characteristics expand the possibilities of action of this virus in the human body ${ }^{3}$. Discoveries about the influence of the virus on the organs, systems, functions, and senses of the human body, as well as the possible impairments after recovery from the COVID-19 condition, are frequent. With regard to hearing and balance, the effects of the virus infection in these systems, secondary disorders caused by it, and alterations due to the use of medications ${ }^{4}$ can be mentioned.

Considering that tissue receptors in the respiratory epithelium are composed of angiotensin-2 converting enzymes (ACE-2), the rapid dissemination of SARS-CoV-2 in the body facilitates its arrival in the highly vascularized cells of the lung. In this case, there is a great possibility that the virus will spread quickly in the body's systems and organs ${ }^{5}$. Possible interactions of this virus with the nervous system can trigger the appearance of sensory disorders, such as smell and taste, as well as vestibular disorder ${ }^{6}$.

The relationship of SARS-CoV-2 with auditory and vestibular alterations can be explained by different hypotheses. Among the possibilities are viral action, the use of ototoxic drugs, local or systemic infections, vascular disorders, and worsening of autoimmune diseases ${ }^{4}$. Thus, this article aims to present preliminary data on the influence of COVID-19 on the appearance and/or worsening of auditory and vestibular symptoms in the population of a Brazilian state.

\section{METHODS}

This research was approved by the Brazilian standards of ethics in research with human beings, by the research ethics committee of the Health Sciences Center of the Federal University of Paraíba, Brazil, and was registered under the number 4,297,779. All participants signed the consent form to participate in the research. This was a cohort study, characterized as observational, descriptive and quantitative approach of research. It was carried out from September 2020 to May 2021 in Paraíba, Northeastern Brazil. The inclusion criteria for the participants were: people with a minimum age of 18 years; test positive for COVID-19; have access to the internet to answer the forms and be in full mental faculties. Participants who were in the acute phase of infection or hospitalized at the time of the research were excluded from the sample, in addition, exposure to noise at work was also an exclusion criterion from the sample. The research consisted of a self-report questionnaire and used the snowball technique, through dissemination in digital media.

The questionnaire used was specially developed for this research by the responsible researchers, being sent through virtual platforms and with mandatory answers to all questions. The questionnaire consisted of 13 questions, which included: age, gender, previous systemic or neurological diseases, previous audiovestibular symptoms, ear surgery, exposure to work noise and use of hearing protection, manifestation of COVID-19, pharmacological treatment, general symptoms after covid, and post-COVID-19 audiovestibular symptoms, their manifestations and duration. All questions were multiple choice, with the exception of the question about the pharmacological treatment, which was an open discursive question.

For statistical data analysis, it was used the SPSS version 22 software and the Chi-Square test for significance analysis with $p<0.05$ as a reference value to difference between pre- and post-covid variables of auditory and vestibular symptoms.

\section{RESULTS}

The sample consisted of 173 people and revealed that most participants were females. The age ranged between 18 and 72 years, with an average age of 35.4 years $(\mathrm{DP}=11.4)$. Regarding the participants who had a previous clinical history of other diseases before infection by COVID-19, the data reveal that respiratory diseases and hypertension were the most mentioned (Table 1). 
Table 1. Pre-existing clinical conditions

\begin{tabular}{lcc}
\hline Previous clinical & N & $\%$ \\
\hline Respiratory diseases & 21 & 12.1 \\
Hypertension & 14 & 8.1 \\
Gastrointestinal Diseases & 11 & 6.3 \\
Endocrinological Diseases & 8 & 4.6 \\
Immunosuppressive Diseases & 6 & 3.4 \\
Diabetes & 5 & 2.8 \\
Neurological Diseases & 5 & 2.8 \\
Cardiopulmonary Diseases / & 4 & 2.3 \\
Cardiovascular & 2 & 1.1 \\
Hematological Diseases & 1 & 0.5 \\
Dermatological Diseases & 1 & 0.5 \\
Muscle Diseases & & \\
\hline
\end{tabular}

Source: the authors

Regarding the manifestation of the disease, most participants reported symptomatic manifestations of COVID-19 with home treatment, followed by asymptomatic, and with a lesser frequency, symptomatic with hospital treatment. The results reveal that the most used pharmacological treatments were Azithromycin and Ivermectin, as shown in Figure 1.

\section{Pharmacological Treatment Used}

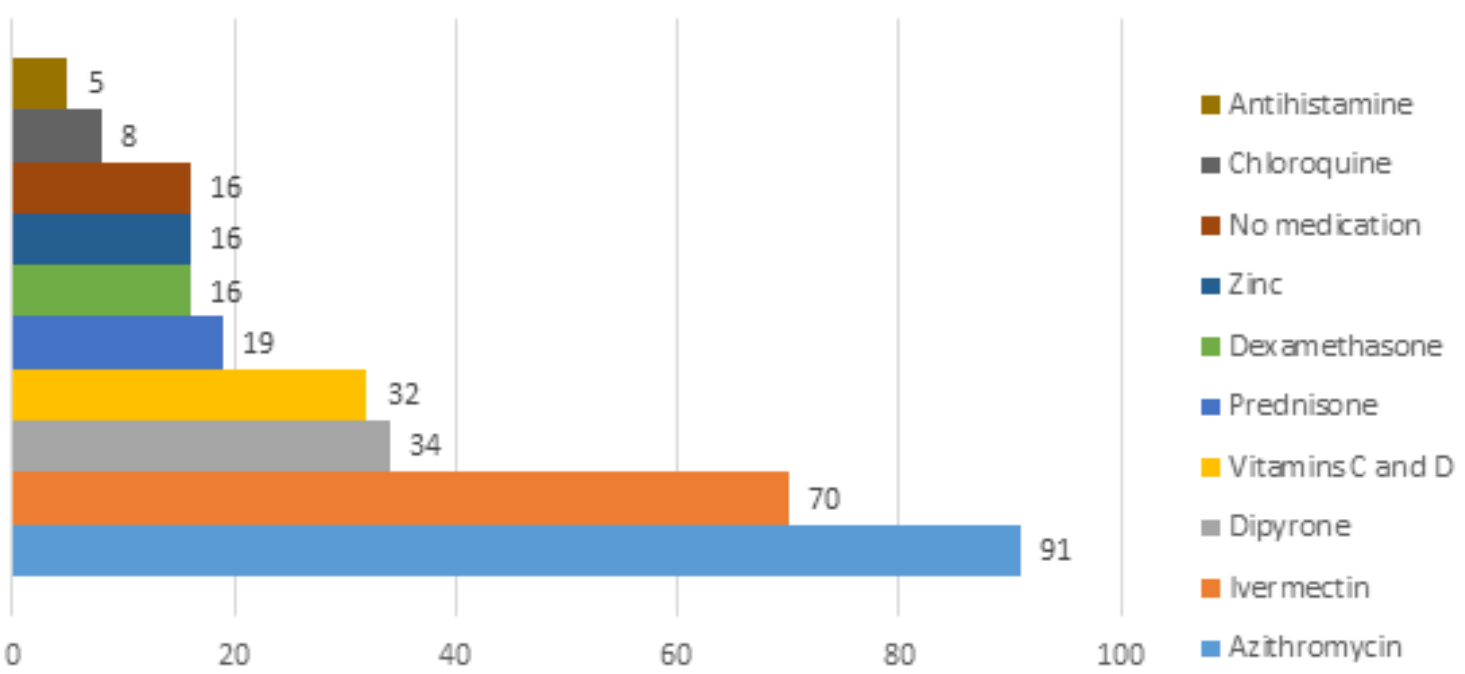

Number of participants

Source: the authors

Figure 1. Pharmacological treatment reported by participants

When asked about extra auditory and vestibular symptoms, the participants mainly reported a decrease in smell, headache, muscle alterations and loss of taste. In addition to these, other symptoms appeared with a lesser frequency, as shown in Table 2. 
Table 2. Extra auditory and vestibular symptoms reported after COVID-19 infection

\begin{tabular}{lcc}
\hline Symptom & N & $\%$ \\
\hline Decreased sense of smell & 126 & 72.8 \\
Headache & 76 & 43.9 \\
Muscle changes & 52 & 30 \\
Loss of taste & 29 & 16.7 \\
Asymptomatic & 17 & 9.8 \\
Skin problem & 16 & 9.2 \\
Smell intolerance & 5 & 2.8 \\
Fatigue & 5 & 2.8 \\
Back pain & 4 & 2.3 \\
Body pain & 2 & 1.1 \\
Mental Confusion & 2 & 1.1 \\
Dry Mouth & 2 & 1.1 \\
Anxiety & 1 & 0.5 \\
\hline
\end{tabular}

Source: the authors

The most reported audio-vestibular symptoms among the participants are tinnitus, dizziness in second place, and ear fullness in third place, as shown in Table 3. Of the 173 participants, $14.5 \%$ reported noticing tinnitus before infection by COVID-19, and this percentage increased to $43.9 \%$ after confirmation of COVID-19. Regarding dizziness, before the coronavirus infection, $11 \%$ of the participants already reported feeling it and this percentage increased to $35 \%$ after the infection. In relation to ear fullness, only $9.2 \%$ reported feeling it before the infection, and this number evolved to $37 \%$ after the confirmation of COVID-19.

Comparing all auditory and vestibular symptoms before and after COVID-19, the results showed significant difference $(p<0.05)$ for tinnitus, dizziness, ear fullness, imbalance, hear but not understand, difficulty hearing in noise and otorrhea (Table 3), Thus, the results showed the occurrence of audio-vestibular symptoms even in those who did not present symptoms before COVID-19.

Table 3. Comparison of self-reported auditory and vestibular symptoms in pre and post COVID-19 infection

\begin{tabular}{lccccc}
\hline \multirow{2}{*}{ Symptom } & \multicolumn{2}{c}{ Before COVID-19 } & \multicolumn{2}{c}{ After COVID-19 } & p-value \\
\cline { 2 - 4 } & $\mathbf{N}$ & \% & N & $\%$ & $<0.001^{*}$ \\
\hline Tinnitus & 25 & 14.5 & 76 & 43.9 & $0.015^{*}$ \\
Dizziness & 19 & 11 & 72 & 35.3 & $0.006^{\star}$ \\
Ear Fullness & 16 & 9.2 & 64 & 37 & $<0.001^{*}$ \\
Imbalance & 14 & 8.1 & 36 & 20.8 & $<0.001^{*}$ \\
Hear but not & 14 & 8.1 & 20 & 11.6 & 0.072 \\
understand & 9 & 5.2 & 17 & 9.8 & 0.123 \\
Hypoacusis & 7 & 4 & 22 & 12.7 & $<0.001^{*}$ \\
Otalgia & 5 & 2.9 & 20 & 11.6 & $<0.001^{*}$ \\
Difficulty Hering in & 5 & 2.9 & 9 & 5.2 & \\
noise & 5 & & & & \\
Otorrhea & & &
\end{tabular}

Source: the authors ${ }^{*} p<0.05$; Chi-square Test 
Regarding the onset and duration of symptoms, $59(34.1 \%)$ participants reported that the symptoms were continuous, with no variation of improvement or worsening, $49(28.3 \%)$ reported the progressively onset, gradually worsening, 43 (24.9\%) reported regressive symptoms, gradually improving, and 22 $(12.7 \%)$ reported immediate onset of these. Regarding persistence, $107(61.8 \%)$ reported that the symptoms remained stable and present so far, and 66 (38.2\%) reported that the manifestation was temporary and disappeared over time.

\section{DISCUSSION}

In the respiratory system, the action of SARS-CoV-2 can trigger the Acute Respiratory Distress Syndrome (ARDS), in addition to contributing to the aggravation of existing conditions ${ }^{7}$. The results showed that among the previously existing diseases, respiratory and hypertension are the most reported. Hypertensive people are more likely to develop severe conditions and higher mortality rate after COVID-19 infection due to the action of the angiotensin 2 converting enzyme (ACE2) in the cardiovascular system ${ }^{8}$.

Extra auditory and vestibular symptoms reported by participants after COVID-19 infection are also presented in other studies ${ }^{9,10}$. Loss of smell and taste is present in $72.8 \%$ and $16.7 \%$ of participants, respectively, and is also described in the study by Scordo et al. $(2021)^{9}$, Yong $(2021)^{10}$ and Vaira et al. (2020) ${ }^{11}$. The authors point out that these most common symptoms of the syndrome may be associated with others, such as headache. In our study, $43.9 \%$ of the participants reported headache and $30 \%$ muscle changes, data that also corroborate the international literature so far $^{9-12}$.

The auditory symptoms investigated in this study point to a possible relationship with SARS-CoV-2, as some participants developed and others worsened the perception of tinnitus, dizziness and hypoacusis. The symptoms investigated showed self-reported increases, with tinnitus highlighted in the report in $43.9 \%$ of the sample after COVID-19, ear fullness in $37 \%$ of the participants and $11.6 \%$ of the sample with difficulty hearing in noise or complaints of hearing and not understand. The worsening or appearance of symptoms may be associated with the drug treatment used (use of ototoxics), inflammation of the auditory and vestibular region due to the virus or also the pandemic situation itself that may exacerbate or trigger such symptoms.
It is noteworthy that medications such as Azithromycin, used as pharmacological treatment in $52.6 \%$ of the study population, may have contributed to a picture of ototoxicity in some cases ${ }^{13}$. Since 1994, hearing loss has been considered a possible side effect of the use of Azithromycin ${ }^{14}$ with potential triggering of tinnitus and dizziness ${ }^{15}$. Taking into account the increase in auditory symptoms after COVID-19 and the percentage of participants who used this medication, a relationship between the drug's ototoxic potential and the appearance of symptoms can be inferred.

The analysis of the results revealed the report of vestibular symptoms after the infection. Dizziness was present in $35.3 \%$ of participants and imbalance in $20.8 \%$. A possible aggravating factor for the appearance of such symptoms may be related to the use of Ivermectin by $40.4 \%$ of participants as treatment. This drug is a potentially toxic drug to the auditory system and especially to the vestibular system ${ }^{15}$, with symptoms such as dizziness and imbalance due to its use being predicted ${ }^{16}$. These data are in line with the questions raised in research by Gallus et al. $(2021)^{17}$, Koumpa et al. (2020) ${ }^{18}$ who investigated hearing and vestibular disorders after COVID-19 infection. The use of other drugs considered harmful to the auditory and vestibular system have also been reported, such as Chloroquine, which can trigger sensorineural hearing loss, vertigo, and tinnitus ${ }^{15}$. Incorrect use and without professional monitoring for this type of medication become a risk factor, which can cause and/or aggravate hearing and vestibular disorders. Therefore, COVID-19 and self-medication may be potential aggravating factors for these conditions.

Some studies have already pointed out possible pathophysiological relationships between the SARS-CoV-2 virus and the appearance and/or worsening of auditory and vestibular symptoms ${ }^{6}$.

These relationships include some hypotheses such as a possible inflammatory process in the cochlea or neuritis caused by viral involvement of the inner ear, or vestibulocochlear nerve ${ }^{19}$. Other hypotheses are crossreactions of antibodies and antigens ${ }^{19}$, cardiovascular manifestations ${ }^{6}$, hypoxia or sequelae and immunemediated diseases ${ }^{20}$. In addition to the reaction such as fighting the virus through the action of drugs ${ }^{15}$.

In early 2020 researches $^{21}$ rarely reported these symptoms, but more recent studies show an increasing prominence of auditory and vestibular symptoms in the population after COVID-196,15,17. In this research, the occurrence of symptoms was observed even 
when it was not reported before COVID. Our results corroborate the respective researches, which leads to several questions regarding the influence of virus mutations, the underreporting of symptoms and the late or long-term effects of the virus on the auditory and vestibular system.

Finally, the report of $61.8 \%$ of the participants about the symptoms remaining stable and present so far, indicates the persistence and possible chronic characteristic of auditory and vestibular symptoms in the population. For this reason, new longitudinal researches must be carried out to enable the monitoring of the population and the definition of strategies for prevention and treatment of these emerging conditions.

One of the limitations of this study is that it was performed in only one Brazilian state. However, the investigation in the national scenario is already underway and aims to characterize the longitudinal behavior and the viral influence on the auditory and vestibular systems. In addition to the possibility of comparisons between different regions of Brazil, there is opportunity of comparing the results with other countries and the investigation of possible local aggravating factors. This study also has a limitation regarding the method of dissemination of the questionnaire, as it is virtual and requires internet access, it may not have reached people of all socioeconomic levels, ages and literacy. Participants in this research were not asked about thetime between answering the questionnaire and the diagnosis of covid-19, which is one of the limitations of the study. The appointment of health care directed to these complaints, as well as instigating the development of new research and intervention strategies for the affected population, is one of our goals. The researchers involved in this study are interested in collaborating with other research centers, with the aim of creating an international database.

\section{CONCLUSION}

The results of this research point to the higher occurrence of auditory and vestibular symptoms, mainly tinnitus and dizziness, after infection by COVID-19. The symptoms are stable and present so far, in most cases.

\section{REFERENCES}

1. WHO. WHO Coronavirus (COVID-19) Dashboard [journal on the internet]. 2021. Covid19.who.int. [cited 7 Jun 2021]. Available at: https://covid19. who.int/
2. Brasil. Coronavírus Brasil: síntese de casos, óbitos, incidência e mortalidade [homepage on the internet]. Ministério da Saúde. 2021. [cited 2021 Jun 7]. Available at: https://covid.saude.gov.br/.

3. Costa VG, Moreli ML, Saivish MV. The emergence of SARS, MERS and novel SARS-2 coronaviruses in the 21st century. Arch. Virol. 2020;165(7):1517-26. https://doi.org/10.1007/s00705-020-04628-0

4. Ciorba A, Corazzi V, Skarżyński PH, Skarżyńska $M B$, Bianchini $C$, Pelucchi $S$ et al. Don't forget ototoxicity during the SARS-CoV-2 (Covid-19) pandemic. Int $\mathrm{J}$ Immunopathol Pharmacol. 2020;34:2058738420941754. https://doi. org/10.1177/2058738420941754

5. Gandhi M, Yokoe DS, Havlir DV. Asymptomatic transmission, the Achilles' heel of current strategies to control Covid-19. N Engl J Med. 2021;38(2):2158-60. https://10.1056/ NEJMe2009758

6. Almufarrij I, Munro KJ. One year on: an updated systematic review of SARS-CoV-2, COVID-19 and audio-vestibular symptoms. Int. J. Audiol. $2021 \mathrm{Mar}$ 2:1-1. https://doi.org/10.1080/14992027.2021.1896 793

7. Aveyard P, Gao M, Lindson N, Hartmann-Boyce J, Watkinson $P$, Young $D$ et al. Association between pre-existing respiratory disease and its treatment, and severe COVID-19: a population cohort study. Lancet Respir. Med. 2021 Apr 1. https://doi. org/10.1016/S2213-2600(21)00095-3

8. Zaki N, Alashwal H, Ibrahim S. Association of hypertension, diabetes, stroke, cancer, kidney disease, and high-cholesterol with COVID-19 disease severity and fatality: a systematic review. Diabetes Metab Syndr. 2020;14(5):1133-42. https:// doi.org/10.1016/j.dsx.2020.07.005

9. Scordo KA, Richmond MM, Munro N. PostCOVID-19 Syndrome: theoretical basis, identification, and management. AACN Adv. Crit. Care. 2021;32(2):188-94. https://doi.org/10.4037/ aacnacc2021492

10. Yong SJ. Long COVID or post-COVID-19 syndrome: putative pathophysiology, risk factors, and treatments. Rev. Infect. Dis. 2021 May 21:1-8. https://doi.org/10.1080/23744235.2021.1924397

11. Vaira LA, Deiana G, Fois AG, Pirina P, Madeddu $G$, De Vito $A$ et al. Objective evaluation of anosmia and ageusia in COVID-19 patients: single-center experience on 72 cases. Head 
neck. 2020;42(6):1252-8. https://doi.org/10.1002/ hed.26204

12. Karaarslan F, Güneri FD, Kardeş S. Postdischarge rheumatic and musculoskeletal symptoms following hospitalization for COVID-19: prospective follow-up by phone interviews. Rheumatol. Int. 2021;41(7):1263-71. https://doi.org/10.1007/ s00296-021-04882-8

13. Rybak LP, Ramkumar V, Mukherjea D. Ototoxicity of Non-aminoglycoside Antibiotics. Front. Neurol. 2021 Mar 9;12:310. https://doi.org/10.3389/ fneur.2021.652674

14. Mick $P$, Westerberg BD. Sensorineural hearing loss as a probable serious adverse drug reaction associated with low-dose oral azithromycin. Am J Otolaryngol. 2007 Oct 1;36(5).

15. Little C, Cosetti MK. A narrative review of pharmacologic treatments for COVID-19: Safety considerations and ototoxicity. Laryngoscope. 2021;131(7):1626-32. https://doi.org/10.1002/ lary. 29424

16. Chandler RE. Serious neurological adverse events after ivermectin - do they occur beyond the indication of onchocerciasis? Am J Trop Med Hyg. 2018;98(2):382. https://doi.org/10.4269/ ajtmh.17-0042

17. Gallus R, Melis A, Rizzo D, Piras A, De Luca LM, Tramaloni $\mathrm{P}$ et al. Audiovestibular symptoms and sequelae in COVID-19 patients. J Vestib Res. 2021(Preprint):1-7. https://doi.org/10.3233/ VES-201505

18. Koumpa FS, Forde CT, Manjaly JG. Sudden irreversible hearing loss post COVID-19. BMJ Case Rep. 2020;13(11):e238419. http://dx.doi. org/10.1136/bcr-2020-238419

19. Lang B, Hintze J, Conlon B. Coronavirus disease 2019 and sudden sensorineural hearing loss. J Laryngol Otol. 2020;134(11):1026-8. https://doi. org/10.1017/S0022215120002145

20. Degen C, Lenarz T, Willenborg K. Acute profound sensorineural hearing loss after COVID-19 pneumonia. Mayo Clin. Proc. 2020;95(8):1801-3. Elsevier. https://doi.org/10.1016/j.mayocp.2020.05. 034

21. Almufarrij I, Uus K, Munro KJ. Does coronavirus affect the audio-vestibular system? A rapid systematic review. Int. J. Audiol. 2020;59(7):487-91. https://doi.org/10.1080/14992027.2020.1776406 